\title{
Fundamentos sobre Alimentación del lactante sano
}

\author{
Healthy infant feeding
}

Agueda Ansela Arriola López*, Belky Xiomara Lazo Calderón**, Jackeline Chinchilla.***

\section{RESUMEN}

La lactancia materna exclusiva durante los primeros seis meses de vida aporta muchos beneficios tanto al niño como a la madre. El inicio temprano de la lactancia materna protege al recién nacido de las infecciones y reduce la mortalidad neonatal. Alrededor de los seis meses, las necesidades de energía y nutrientes del lactante empiezan a ser superiores a lo que puede aportar la leche materna, por lo que se hace necesaria la introducción de alimentos complementarios. Si no se introducen alimentos complementarios alrededor de los seis meses o si son administrados de forma inadecuada, el crecimiento del niño puede verse afectado. Por lo que el objetivo de este artículo es revisar los fundamentos sobre la alimentación del lactante.

\section{PALABRAS CLAVE}

Lactante, lactancia materna, ablactación, alimentación complementaria.

\section{ABSTRACTS}

Exclusive breastfeeding during the first six months of life brings many benefits for both the child and the mother. Early initiation of breastfeeding protects the newborn from infection and it reduces neonatal mortality. Around six months of age, the infant's energy and nutrient requirements increases, therefore the introduction of complementary feedings is necessary. If complementary foods are introduced around six months or if administered improperly, the child's growth may be affected. The purpose of this article is to review the fundamentals of infant feeding.

*Médico Residente Segundo Año. Postgrado Pediatría. Universidad

Nacional Autónoma de Honduras Valle de Sula. UNAH-VS

**Médico Residente Tercer Año. Postgrado Pediatría. UNAH-VS.

*** Médico Especialista en Pediatría. HNMCR

Dirigir correspondencia a: aaarriola_lopez@hotmail.com

Recibido: 16 de junio 2016 Aprobado: 20 de agosto 2016

\section{KEY WORDS}

Infant, breastfeeding, weaning , complementary feeding.

\section{INTRODUCCION}

Una adecuada nutrición durante la infancia y niñez temprana es esencial para asegurar que los niños alcancen todo su potencial en relación al crecimiento, salud y desarrollo. La falta de acceso a alimentos asequibles y ricos en nutrientes es un problema constante para muchas familias en todo el mundo. Con mucha frecuencia se introducen alimentos sólidos y blandos demasiado pronto o demasiado tarde, la frecuencia y la cantidad de comida que se ofrece es menor de lo que se necesita para el crecimiento normal del niño. ${ }^{(1)}$

En la presente revisión abordaremos en que consiste la alimentación del lactante sano, períodos de la alimentación infantil, funciones digestivas del lactante, períodos de la leche humana, contraindicaciones de la lactancia materna, ablactación y los alimentos sugeridos por edad.

\section{ALIMENTACIÓN DEL LACTANTE SANO}

La palabra lactante, del latín lactantes, define, en su primera acepción, al que mama, o sea, la criatura en el período de lactancia; niño de pecho. La leche de pecho es el único alimento necesario para cubrir sus necesidades de crecimiento y desarrollo adecuados hasta los 6 meses de vida. Cualquier otro alimento representará, desde el punto de vista fisiológico, una agresión para su organismo. ${ }^{(2)}$

La etapa de lactante se extiende desde el momento del nacimiento hasta los dos años de edad. A lo largo de este periodo, el niño va a comenzar su alimentación con la ingesta exclusiva de leche, ya que su inmadurez solo le permite la utilización de sus reflejos primarios 
de succión y deglución. Posteriormente, en las siguientes etapas, podrá incorporar los alimentos semilíquidos y después los sólidos. A lo largo de estos dos años se van a producir fenómenos madurativos importantes que afectan al sistema nervioso, al aparato digestivo y al aparato excretor, por lo que la alimentación debe ser la adecuada a cada edad y etapa madurativa. Debe ir adaptándose a nuevos sabores y texturas y familiarizarse con la forma de presentación de la comida de los adultos. ${ }^{(3)}$

\section{PERÍODOS DE LA ALIMENTACIÓN INFANTIL}

El lactante es el niño que se alimenta fundamentalmente de leche. Los "Períodos de la Alimentación del Niño" como definió el Comité de Nutrición de la Academia Americana de Pediatría en 1982 son tres:

- Período de lactancia: comprende los 6 primeros meses de vida, durante los cuales su alimento debe ser de forma exclusiva la leche materna (según la OMS), y en su defecto, las fórmulas para lactantes.

- Período transicional: integra el segundo semestre de vida, hasta cumplir un año. En él se inicia la Diversificación Alimentaria que comporta el inicio progresivo de la alimentación complementaria, introduciendo alimentos distintos a la leche materna o fórmula.

- Período de adulto modificado: abarca la edad preescolar y escolar hasta los 7-8 años de edad. En este período el niño va adoptando una alimentación progresivamente más parecida a la de los adultos, y gradualmente a una dieta que proporcione un $30 \%$ de la energía total en forma de grasa, y de ésta un tercio en forma de grasa saturada, en lugar del $50 \%$ de grasa y mayor proporción de grasa saturada propia de la leche materna. ${ }^{(3,4)}$

\section{FUNCIONES DIGESTIVAS DEL LACTANTE}

El lactante sano es capaz de una succión efectiva, con reflejo del cierre anatómico de la glotis. Sin embargo, la deglución de sólidos es impedida por movimientos de extrusión de la lengua hasta el $4^{\circ}$ o $5^{\circ}$ mes de vida. Los movimientos masticatorios reflejos aparecen entre el séptimo y noveno mes de vida, aunque no tenga dientes. En la saliva tanto la amilasa, presente antes que la amilasa pancreática, y la lipasa lingual, están bien desarrolladas al nacimiento e inician la hidrólisis de los triglicéridos de la leche. El tono del esfínter esofágico inferior aumenta progresivamente en los 6 primeros meses, aunque su completa madurez se alcanza a los 3 años. ${ }^{(4)}$

Las pautas de vaciado gástrico pueden verse influidas por el contenido proteico y graso del alimento. La leche materna se vacía en dos fases, una primera rápida y otra lenta. La leche de fórmula se vacía más lentamente y de forma lineal. El ritmo de vaciado gástrico normal se alcanza hacia los 9 meses de edad. El pH gástrico es más alto que el del adulto; alcanza los valores de este hacia los 3 años de edad. La secreción de pepsina es baja hasta los 3 meses, y hasta los 18 meses alcanza valores del adulto. Al ser el pH gástrico menos ácido, la acción de la pepsina sobre la digestión de proteínas es menor, lo que puede favorecer el paso a la circulación de proteínas enteras. La secreción del factor intrínseco es la mitad que la del adulto hasta los 3 meses, pero el lactante pequeño es capaz de absorber la vitamina B12 por un mecanismo distinto a este.

Al mes de edad el volumen de secreción pancreática es normal. La actividad amilasa es nula al nacimiento y va aumentado hasta los 3 años; es inducible por el sustrato como por ejemplo, al dar almidón. La actividad de tripsina quimotripsina y lipasa, están presentes desde el nacimiento $y$, la respuesta a la secretina desde el primer mes. La secreción de sales biliares es insuficiente hasta el primer mes de vida, y la concentración micelar crítica es menor. ${ }^{(4)}$

Morfológicamente el intestino está maduro, pero bioquímicamente al nacimiento la lactasa y maltasa son un tercio de los valores del adulto. Los sistemas de absorción están presentes pero la bomba sodio-potasio no alcanza valores normales hasta el año de edad. Las enzimas citoplasmáticas a nivel de enterocitos funcionan bien ya al nacimiento. La tolerancia inmunológica viene marcada por el tipo de proteínas, digestión y momento de contacto con la pared intestinal. La barrera intestinal constituye una defensa 
contra numerosas agresiones antigénicas: alimentarias, bacterianas, víricas y parasitarias. La hipoacidez gástrica del lactante pequeño, disminución de sales biliares y motilidad, pueden contribuir al contacto con dichos antígenos en un momento en el que no está bien desarrollado el sistema linforreticular asociado al intestino, y la introducción de proteínas heterólogas podrán ser fuente de intolerancia o alergia cuanto más precozmente se introduzcan. ${ }^{(4)}$

Otra función que tiene que alcanzar su madurez en el primer año es la renal. En los tres primeros meses, el lactante alcanza una filtración glomerular que le permite mayor tolerancia al agua y solutos, pero los valores del adulto no se alcanzan hasta los 2 años. Son bajos también los valores de excreción y reabsorción tubular. Pero si la alimentación es adecuada el lactante puede tener una función renal satisfactoria. Es capaz de diluir la orina, siempre que no se le administren cantidades excesivas de líquidos hipertónicos. Tiene menos capacidad de concentración renal por ser más cortas las asas de Henle, bajo transporte tubular de sodio, mayor flujo medular sanguíneo, baja excreción de urea y menor respuesta tubular a la hormona antidiurética. Es muy importante tener en cuenta que el lactante no dispone de ningún sistema de excreción de sodio, y éste se controla variando la reabsorción tubular del sodio filtrado. La ingesta moderada de sodio el lactante la tolera bien, pero eliminar un exceso de sodio, puede acarrearle un grave problema del medio interno. Se estiman unas necesidades diarias de sodio de 2-3 mEq/100 Kcal metabolizadas o 1-1,5 $\mathrm{mEq} / \mathrm{kg} / \mathrm{día}$. Si el lactante recibe alimentos con elevada carga de solutos sin suplemento de agua, puede presentar un balance hídrico negativo. Lo que podría ocurrir con fórmulas distintas a la leche materna que no estuvieran adaptadas o con la introducción precoz de alimentos sólidos en la dieta. Por tanto, hay que tener muy en cuenta, que los riñones maduran morfológica y funcionalmente durante el primer año de la vida. ${ }^{(4)}$

El proceso de maduración del sistema nervioso central también va marcando los distintos períodos de la alimentación del niño. La madu- ración del sistema neuromuscular hasta los 4 meses permite deglutir líquidos. De los 4-6 deglutirá semisólidos y posteriormente adquirirá la masticación. La sedestación a partir de los 6 meses y luego la bipedestación le permitirán distinguir objetos, colores, coger y manipular las cosas y elegir incluso alimentos. ${ }^{(4,5)}$

\section{LA LECHE HUMANA}

La lactancia materna (LM) es una de las estrategias más costo efectivas para prevenir la morbilidad y mortalidad infantil. La lactancia natural tiene beneficios para el lactante tanto como para la madre; se asocia con menor riesgo de sufrir cáncer de mama o de ovarios, y de diabetes en la mujer. Y está relacionada con ahorros familiares sustanciales asociados con la compra de fórmula. ${ }^{(6,7)}$

La leche humana es un fluido biológico complejo que contiene proteínas, nitrógeno no proteico, hidratos de carbono, lípidos, vitaminas hidrosolubles, minerales, iones y células, y que presenta una serie de propiedades nutricionales que aventajan a la leche de vaca para la alimentación del niño en este período. Es un líquido dinámico que cambia cronológicamente, diferenciándose la composición con la edad del bebé. ${ }^{(3)}$

El calostro es la leche que se produce desde el nacimiento hasta el $4^{\circ}-6^{\circ}$ día de vida. Posee gran contenido proteico, IgA secretora, lactoferrina, oligosacáridos, factor de crecimiento intestinal y minerales. Es pobre en grasa y predomina en ella el colesterol. Su función principal es la de proporcionar lo que el niño necesita para el crecimiento y protección del aparato digestivo.

La leche de transición (desde el $6^{\circ}$ al $15^{\circ}$ día de vida del bebé) tiene una composición intermedia entre el calostro y la leche madura. En su composición disminuyen la cantidad de inmunoglobulinas, aumenta la lactosa, los lípidos, las vitaminas liposolubles e hidrosolubles.

La leche madura tiene un contenido energético mayor $(700 \mathrm{Kcal} / \mathrm{L})$. El $80 \%$ es agua, con un contenido proteico de 0,9-1,2 g/dl, más bajo que la leche de vaca $(3,5 \mathrm{~g} / \mathrm{dl}) .(3)$ 
La primera semana es esencial para el éxito de la lactancia. Es conveniente favorecer la relación madre-hijo inmediatamente tras el parto e iniciar la lactancia tan pronto como el estado físico de la madre y el hijo lo permita, a ser posible antes de las 6 horas. Durante los primeros días, la madre necesitará ayuda para vencer los inconvenientes que se presentan durante la lactancia, como llanto, somnolencia, regurgitaciones del bebé, pezones sensibles, dolor por la episiotomía, depresión postparto e inseguridad en el éxito de la lactancia. Los primeros días las tomas deben ser cada 2 horas para ir espaciándolas en semanas posteriores hasta llegar a cada 3-4 horas. La mayoría de la leche la obtiene el niño al principio de la tetada, es decir, el $50 \%$ en los primeros 2 minutos y el $80-90 \%$ en los primeros 7-8 minutos. $^{(8)}$

\section{Tabla No. 1: Contraindicaciones de la Lactancia Materna.}

\section{Contraindicaciones absolutas}

Cáncer materno/quimioterapia

Tuberculosis

Madre drogadicta

Fármacos maternos

Metabolopatias: Galactosemia

Malformaciones

Madre con psicosis grave

\section{Contraindicaciones relativas}

Estreptococo B neonatal

Infecciones maternas agudas

Enfermedades orgánicas graves

Virosis: (HBV, CMV, VIH, HTLV-1)

Fibrosis quística

Psicopatías maternas

Epilepsia materna no controlada

Fuente: Manual Práctico de Nutrición en Pediatría. Comité de Nutrición de la Asociación Española de Pediatría.

\section{De 0 a 4 meses}

La lactancia materna o fórmula de inicio 1 (leche 1) está indicada para cubrir todas las necesidades del lactante hasta los 4-6 meses de vida. Se administrará cada 3-4 horas. La madre debe lavarse las manos con agua y jabón antes de cada mamada. Debe limpiar el pezón y la areola cuidadosamente con agua hervida antes y después de darle el pecho a su hijo. Debe sentarse cómodamente, con la espalda bien apoyada y recta, el ambiente tranquilo, con la luz adecuada y, si es posible, en el mismo lugar de la casa. El bebé debe estar en posición semivertical y la madre debe comprimir el pecho alrededor del pezón con los dedos pulgar e índice para que la nariz del niño quede libre. Una buena técnica de lactancia materna consistirá en dar un pecho durante unos 7-8 minutos y pasar al segundo hasta que el niño se canse y quede satisfecho. En la siguiente mamada se empezará por este último y así sucesivamente. La costumbre de mantener el bebé al primer pecho durante 10-15 o más minutos, mientras el otro pecho rebosa de leche, solo conduce a que el bebé se canse y trague aire, con lo que se provoca meteorismo y cólicos del lactante. ${ }^{(8,9)}$

Durante el período de lactancia la madre debe eliminar de su alimentación:

1. Bebidas estimulantes: café, té, bebidas con cola, chocolate y cacao.

2. Bebidas alcohólicas, incluidas las denominadas sin alcohol. El alcohol ingerido pasa a la leche materna y puede perjudicar al niño.

3. Vegetales: coliflor, col, alcachofas, espárragos, pepino, pimientos, ya que pueden cambiar el sabor natural de la leche materna.

4. Medicamentos: solo puede tomar los estrictamente recomendados por su médico. ${ }^{(8)}$

\section{ALIMENTACIÓN COMPLEMENTARIA}

La alimentación complementaria debe aportar una densidad energética no inferior a la aportada a través de la leche materna y el volumen de esta debe ir aumentando en forma progresiva de acuerdo a la edad y aceptabilidad del lactante. Es importante destacar que es en el período de los 6 a 24 meses donde se establecen la mayor parte de los hábitos, preferencias y aversiones alimentarias que condicionarán el tipo de alimentación futura. ${ }^{(9)}$

El inicio de la alimentación complementaria además depende de la madurez morfofuncional del niño: digestión y absorción adecuada de nutrientes, control de cabeza (capacidad de 
levantar y sostener la cabeza, en general a los 2 meses) y de tronco (capacidad de sentarse sin apoyo, en promedio a los 6 meses), uso de la musculatura masticatoria, erupción dentaria, incremento de las percepciones sensoriales (olfato, visión, gusto y tacto de alimentos), extinción del reflejo de extrusión y discriminación de nuevas texturas, sabores, olores, temperaturas y consistencia de los alimentos. ${ }^{(10-13)}$

La alimentación complementaria guiada por el bebé (baby-ledweaning) se basa en que sea el propio lactante quien se alimenta llevándose la comida a la boca, en vez de ser alimentado con una cuchara por un adulto. En esta práctica, el lactante se incorpora pronto a la comida familiar y comparte su menú, manteniendo la lactancia materna. Algunos estudios observacionales de pequeño tamaño sugieren que esta técnica favorece los patrones de alimentación, aunque no se ha podido demostrar si se plasma en efectos beneficiosos para la salud. Su difusión surge a raíz de la recomendación de la Organización Mundial de la Salud de retrasar la introducción de la alimentación complementaria hasta los 6 meses, en un momento en que el lactante ha alcanzado hitos importantes en su desarrollo, lo que haría posible que se alimentara por sí mismo. ${ }^{(14)}$

La Asociación Española de Pediatría (AEP), la Sociedad Europea de Gastroenterología, Hepatología y Nutrición Pediátrica (ESPGHAN), la Sociedad Española de Gastroenterología, Hepatología y Nutrición Pediátrica (SEGHNP) y la Academia Americana de Pediatría (AAP) recomiendan la lactancia materna como el mejor alimento del recién nacido y lactante durante los primeros 4-6 meses de vida, coincidiendo en 3 puntos básicos:

1. La leche materna es el mejor alimento para el recién nacido y lactante.

2. Si no es posible aconsejan una leche adaptada o fórmula infantil.

3. La alimentación complementaria debe iniciarse a partir del $4^{\circ}$ mes de vida valorando previamente la maduración del niño y su estado nutricional. $(8,15)$

Los lactantes son particularmente vulnerables durante el período de transición en el que comienza la alimentación complementaria. Por lo tanto, para asegurarse de que se satisfacen sus necesidades nutricionales, los alimentos complementarios tienen que cumplir los requisitos siguientes:

- Han de ser oportunos, es decir, se deben introducir cuando las necesidades de energía y de nutrientes sobrepasan lo que puede proporcionarse mediante la lactancia natural exclusiva y frecuente;

- Han de ser adecuados, es decir, deben proporcionan energía, proteínas y micronutrientes suficientes para satisfacer las necesidades nutricionales de un niño en crecimiento;

- Han de ser inocuos, es decir, se deben preparar y almacenar de forma higiénica y hay que darlos con las manos limpias y utilizando utensilios limpios, y no biberones y tetinas;

- Han de darse de forma adecuada, es decir, se deben dar atendiendo a las señales de apetito y de saciedad del niño, y la frecuencia de las comidas y el método de alimentación deben ser adecuados para su edad (alentar activamente al niño a que, incluso cuando está enfermo, consuma alimentos suficientes utilizando los dedos, una cuchara o alimentándose por sí mismo). ${ }^{(13,16)}$

\section{De 4 a 5 meses}

A partir del $4^{\circ}$ mes de vida se inicia la papilla de frutas (por la tarde) que corresponderá a una merienda con aporte de vitaminas naturales y fibra, que son importantes para la salud del niño. Tras la papilla de frutas a los pocos días pueden añadirse a 1 ó 2 biberones al día unas cucharaditas de harina sin gluten. ${ }^{(8)}$

\section{De 5 a 6 meses}

De los 5 a 6 meses y medio de edad se inicia la primera papilla salada en la alimentación del bebé que consiste en un puré de verduras de pollo. Se inicia con 60- 70 gramos de pollo para ir aumentando en los siguientes días hasta los100-120 gramos. Paralelamente se van reduciendo el número de tomas por día y se puede cambiar la fórmula de inicio (leche 1) por una leche de continuación (leche 2 ). Una vez cumplido el $6^{\circ}$ mes de vida se amplía la variedad de harina, escogiéndose entre las que contienen gluten. ${ }^{(8)}$ 


\section{De 7 a 8 meses}

Entre el $7^{\circ}$ y $8^{\circ}$ mes se introduce la carne de ternera alternándola con el pollo en la toma del mediodía. A partir del $8^{\circ}$ mes se inicia una papilla salada por la noche con la introducción del pescado blanco (rape, merluza, lenguado) 3-4 veces a la semana. Con la introducción del pescado se inicia el aporte de aceites de pescado de gran interés para un correcto equilibrio entre la grasa saturada (de origen animal) y poliinsaturada (de origen vegetal y marino). El aporte de leche diario debe ser aproximadamente de 500 cc durante la infancia. ${ }^{(8)}$

Al cenar el niño el pescado, en los casos que sólo haga 4 tomas al día, el aporte de leche se vería mermado si no se aplica algún cambio en su alimentación. El aporte de leche en esta edad todavía es básico para un buen desarrollo, por lo que no debe ser inferior a los 500 cc/día. Para ello, el día en que se desee darle pescado al bebé se suprimirá la papilla de frutas y se administrará una papilla con leche 2 y cereales igual a la del desayuno. ${ }^{(8)}$

\section{De 10 a 12 meses}

A los 10-12 meses de vida se introduce el huevo para la cena alternando con el pescado una vez a la semana. Primero será huevo duro y solo la yema; pasadas 2-3 semanas ya puede administrarse completo. En forma de tortilla francesa se iniciará a los 12-15 meses dependiendo de la habilidad del bebé para la masticación. ${ }^{(8)}$

\section{De 12-24 meses}

La consistencia de los alimentos debe ser molida desde los 12 meses y picados desde los 18 meses. Es aconsejable a esta edad introducir el consumo de verduras crudas y estimular la ingestión de vegetales. Por otro lado, no es recomendable el consumo de golosinas (helados, dulces, chocolates, galletas, bebidas gaseosas, jugos en polvo, concentrados lácteos, papas fritas, cereales azucarados) y se debe restringir el aporte adicional de sal ya que en este período el niño está formando sus hábitos y preferencias. ${ }^{(5)}$ Desde la perspectiva de la obesidad, se ha buscado la relación entre la alimentación en el primer año de vida y la obesidad infantil. Es común denominador la tendencia a que los patrones adoptados propicien la obesidad al hacer uso de alimentos y bebidas adipogénicas, tal como se expuso en la Conferencia sobre la Prevención dela Obesidad Infantil en 2003 de la Academia Americana de Pediatría, en donde se reportó que este tipo de prácticas, con exposición del menor a alimentos ricos en contenido energético pero pobres en valor nutritivo, fueron empleadas muy frecuentemente y en edades tempranas ( 7 meses de edad). ${ }^{(17)}$

\section{Tabla No. 2: DECÁlOGO DE UNA BUENA ALIMENTACIÓN DEL LACTANTE.}

1. Lactancia materna, exclusiva hasta el 4-6 mes.

2. Continuar la lactancia materna durante el período de ablactación hasta los 2 años de edad.

3. Fórmula de inicio (leche 1) hasta el 4-6 mes si no es posible la lactancia materna.

4. Fórmula de continuación (leche 2) a partir del 4-6 meses y hasta los 2-3 años. De los 6 a los 12 meses el aporte de leche debe ser de 500 cc al día. La leche de vaca entera y los derivados se ofrecerán después de los 12 meses.

5. Iniciar el gluten después del $6^{\circ}$ mes.

6. Aporte de 400 UI/día de vitamina D.

7. Secuencia de introducción de alimentos:

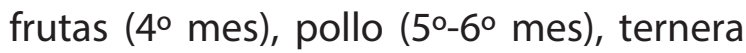
(6०-70 mes), pescado ( $\left.8^{\circ} \mathrm{mes}\right)$, huevo-yema

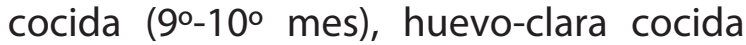
(11 ${ }^{\circ}-12^{\circ}$ mes).

8. No introducir en la alimentación ningún alimento sin el consejo de su pediatra. Los cambios de alimentación e introducción de nuevos alimentos se harán poco a poco. El cambio de un sabor a otro requiere a veces ofertas repetidas para su aceptación.

9. Alimentos no recalentados ni preparados más de 8-12 horas antes de ser consumidos por el niño.

10. Tener cuidado extremo con las medidas higiénicas durante el primer año de vida e incluso "esterilizar" durante los primeros 6 meses.

Fuente: Manual Práctico de Nutrición en Pediatría. Comité de Nutrición de la Asociación Española de Pediatría. ${ }^{(8)}$ 
La introducción de alimentos sólidos antes de los 4 meses de edad en niños que nunca recibieron lactancia materna o la recibieron menos de 4 meses en comparación con los que la recibieron después de esta edad, aumentó en 6 veces el riesgo de obesidad a los 3 años de edad, independientemente de la velocidad de crecimiento. ${ }^{(18-19)}$

\section{ALIMENTOS ALERGÉNICOS}

La edad a la que los alimentos alergénicos, como maní, huevo cocido, leche de vaca, sésamo, pescado blanco y el trigo, deben ser introducidos en la dieta de lactancia materna infantes aún es incierto. ${ }^{(20)}$

\section{CONCLUSION}

La lactancia materna es y seguirá siendo el alimento más seguro y costo efectivo para los lactantes, dado los múltiples beneficios ya conocidos de la leche materna, además de que esta disminuye el riesgo de morbi-mortalidad de millones de niños anualmente alrededor del mundo; la alimentación complementaria debe iniciarse a los 4-6 meses según la condición y las exigencias del lactante, ya que a partir de esta edad la lactancia materna resulta insuficiente para suplir los requerimientos dietéticos requeridos para lograr un crecimiento y desarrollo adecuados, por lo que es de vital importancia adquirir el conocimiento en cuanto a la correcta introducción de la alimentación complementaria según edad y tipo de alimento, para garantizar el desarrollo de niños sanos.

\section{BIBLIOGRAFÍA}

1. UNICEF. Lactancia materna y alimentación complementaria http://www.unicef.org/spa nish/nutrition/index_breastfeeding.html.

2. Díaz-Argüelles Ramírez-Corría VM. La alimentación inadecuada del lactante sano y sus consecuencias. Rev Cubana Pediatr [Internet]. 2005 Mar [citado 2016 junio 10]; 77 (1): Disponible en: http://scielo.sld. $\mathrm{cu} /$ scielo.php?script $=$ sci_arttext $\&$ pid $=$ S0034-5312005000100007\&lng=es Pavón P. Parra I. Aparicio M. Arroba M.

3. Comité de Nutrición de la Asociación Española de Pediatría. Manual Práctico de Nutri ción en Pediatría. Majadahonda: ERGON; 2007.

4. Lázaro Almazar A, Martínez BM. Alimentación del lactante sano. En: SEGNP-AEP. Protocolos diagnóstico terapéuticos de gastroenterología, hepatología y nutrición pediátrica. Majadahonda: ARGON; 2008. p. 287-295.

5. Torrejón SC, Osorio J, Vildoso FM, Castillo DC. Alimentación del niño menor de 2 años: Recomendaciones de la Rama de Nutrición de la Sociedad Chilena de Pediatría. Rev. chil. pediatr. [Internet]. 2005 [citado 2016 Jun 05] ; 76( 1 ): 91-97. Disponible en: http://www.scielo.cl/scielo. php?script=sci_arttext\&pid= S0370-41062 005000100013.

6. González de Cosío T, Escobar-Zaragoza L, González-Castell L D, Rivera-Dommarco JA. Prácticas de alimentación infantil y deterioro de la lactancia materna en México. Salud pública Méx [Internet]. 2013 [citado 2016 Jun 05] ; 55( Suppl 2 ): S170-S179. Disponible en: http://www.scielo.org.mx/scielo. php? script =sci_arttext\&pid=S00 36-36342013000800014\&lng=es.

7. Stuebe, AM. Formas de facilitar que la mujer alcance sus metas de lactancia materna. Obstet Gynecol 2014;123:64352.

8. Acuña M. Alonso M. Álvarez-Coca J. Arguelles F. Armas H. Aznal E. et al. Tratamiento en gastroenterología, hepatología y nutrición pediátrica. 2a . Ed. España: SEGHNP. 2008. 
9. Muñoz E., Casanello, P, Krause B, Uauy R. La alimentación de la madre, el bebé y el niño. Mediterráneo económico. 2015; (27):57-74.

10. Castillo-Durán C. Balboa C. Torrejón C. Bascuñán K. Uauy R. Alimentación normal del niño menor de 2 años: Recomendaciones de la Rama de Nutrición de la Sociedad Chilena de Pediatría 2013. Rev. chil. pediatr. [Internet]. 2013 [citado 2016 Jun 10]; 84 (5): 565-572. Disponible en: http://www. scielo.cl/scielo.php?script=sci_arttext\&pi $d=$ S0370-41062013000500013.

11. Nwaru BI, Takkinen HM, Niemelä O, Kaila M, Erkkola M, Ahomen S, et al. Introduction of complementary foods in infancy and atopic sensitization at the age of 5 years: timing and food diversity in a Finnish birth cohort. Allergy 2013; 68(4): 507-16.

12. Cattaneo A, Williams $C$, Pallás-Alonso $C R$, Hernández-Aguilar MT, Lasarte-Velillas JJ, Landa-Rivera L, et al. ESPGHAN's 2008 recommendation for early introduction of complementary foods: how good is the evidence? Matern Child Nutr 2011; 7(4): 335-43.

13. Santos Prieto $D$, Mai Thu $Q$, Véliz Concepción OL, Grau Ábalo R, Hurtado Aguilar L. Mature deglutition in children aged 2 to 5 years and their eating habits. Medicentr Electrón [Internet]. 2016 [citado 2016 Jun 05] ; 20( 2 ): 104-111. Disponible en: http://www.medigraphic.com/pdfs/medicentro/cmc-2016/cmc162c.pdf.

14. González de Cosío T. Escobar-Zaragoza L. González-Castell LD. Rivera-Dommarco JA. Prácticas de alimentación infantil y deterioro de la lactancia materna en México. Salud pública Méx [Internet]. 2013 [citado 2016 Jun 05] ; 55( Suppl 2 ): S170-S179.

Disponible en: http://www.scielo.org.mx/ scielo.php?script=sci_arttext\&pid= S0036-36342013000800014\&lng=es.
15. Villares JM, Galeano Segovia MJ, Dalmau Serra J. Alimentación complementaria dirigida por el bebé («baby-led weaning»). ¿Es una aproximación válida a la introducción de nuevos alimentos en el lactante? Acta Pediatr Esp 2013; 71(4), 99-103.

16. Organización Mundial de la Salud. Estrategia Mundial para la Alimentación del Lactante y del Niño Pequeño. Ginebra: OMS; 2003.

17. Izquierdo E, Segoviano Lorenzo C, Vergues Penia C. Alimentación Complementaria: que, cuando y como. Rev pediatr Aten Primaria [internet] . 2016 [citado jun 12 2016];18:e31-e35. Disponible en: http:// www.pap.es/files/1116-2069-pdf/08_RPAP _1065_Alimentacion-complementaria. pdf.

18. Sandoval Jurado $L$, Jiménez Báes MV, Olivares Juárez $\mathrm{S}$, Olvera T. Lactancia materna, alimentación complementaria y el riesgo de obesidad infantil. Aten Primaria. [Internet] 2016 [citado jun 12 2016];49(9): 572-578. Disponible en: http://dx.doi. org/10.1016/j.aprim.2015.10.004

19. Macías-Tomei C, Herrera Hernández $M$, Mariño Elizondo M, Useche Daniela. Crecimiento, nutrición temprana en el niño y riesgo de obesidad. Arch Venez Puer Ped [Internet]. 2014 [citado 2016 Jun 10]; 77(3): 144-153. Disponible en: http:// www.scielo.org.ve/scielo.php?script $=$ s ci_arttext\&pid=S00040649201400030000 $8 \& \operatorname{lng}=$ es.

20. Perkin MR, Logan K, Tseng A, Raji B, Ayis S, Peacock J, et al. Randomized Trial of Introduction of Allergenic Foods in Breast-Fed Infants. N Engl J Med 2016; 3749(18): 1733-43. 